\title{
To study heterogeneity in SARS-CoV-2 IgG response titre in patients recovered from COVID-19
}

\author{
Avneet Garg1, Rakendra Singh², Hariharan Iyer ${ }^{3}$, Mansimranjit Kaur ${ }^{1}$, Surbhi ${ }^{2}$, Ashish Jindal'2, \\ Saranpal Singh $^{4}$, Avtar Singh Bansal ${ }^{5}$, Hem C Sati ${ }^{6}$, Vinita Jindal ${ }^{7}$
}

${ }^{1}$ Department of Respiratory Medicine, Adesh institute of Medical Sciences and Research, Bathinda; ${ }^{2}$ Department of Medicine, Adesh institute of Medical Sciences and Research, Bathinda; ${ }^{3}$ Department of Pulmonary Medicine, Critical Care and Sleep Medicine, All India Institute of Medical Sciences, New Delhi; ${ }^{4}$ Department of Biochemistry, Adesh institute of Medical Sciences and Research, Bathinda; ${ }^{5}$ Department of Community Medicine, Adesh institute of Medical Sciences and Research, Bathinda; 'Department of Biostatics, Critical Care and Sleep Medicine, All India Institute of Medical Sciences, New Delhi; ' Department of Radiology, Adesh institute of Medical Sciences and Research, Bathinda, India

\begin{abstract}
Correspondence: Dr. Vinita Jindal, MBBS, MD, Assistant Professor, Adesh Institute of Medical Sciences and Research (AIMSR),

Bathinda, Punjab, 310, B-faculty block, AIMSR, Bathinda, Punjab, India.

Tel. +91.09888444698 .

E-mail: dravneetgarg@gmail.com

Keywords: COVID-19; SARS-CoV-2-IgG antibodies; CT severity score; age.

Acknowledgments: We acknowledge all the health care workers and students for participating in the study

Contributions: AG, conception and design of work, acquisition, analysis, interpretation of data, drafting and revising the work; RS, conception and design of work, drafting and revising the work critically; HI, S, acquisition, analysis, interpretation of data, drafting and revising the work; MK, acquisition of data, drafting the work, revising the work critically; AJ, SS, ASB, acquisition of data, drafting and revising the work; HCS, conception and design of work, analysis, interpretation of data, drafting and revising the work; VJ, conception and design of work, acquisition of data, drafting and revising the work. All the authors have read and approved the final version of the manuscript and agreed to be accountable for all aspects of the work ensuring that questions related to the accuracy or integrity of any part of the work are appropriately investigated and resolved.
\end{abstract}

Conflict of interest: The authors declare that they have no competing interests, and all authors confirm accuracy.

Ethical approval: Permission obtained from the Institutional Research and Ethical Committee for conducting the study.

Informed consent: Obtained from all the participants.

Received for publication: 23 May 2021.

Accepted for publication: 12 August 2021

COpyright: the Author(s), 2021

Licensee PAGEPress, Italy

Monaldi Archives for Chest Disease 2022; 92:1943

doi: 10.4081/monaldi.2021.1943

This article is distributed under the terms of the Creative Commons Attribution Noncommercial License (by-nc 4.0) which permits any noncommercial use, distribution, and reproduction in any medium, provided the original author(s) and source are credited.

\begin{abstract}
The COVID-19 pandemic caused a serious health challenge to the entire mankind. The association between clinical characteristics of disease and formation of neutralizing antibodies have not well studied. A prospective study was conducted for patients recovered from confirmed SARS-CoV-2 infections from $1^{\text {st }}$ August 2020 to $28^{\text {th }}$ February 2021, to study the association between SARS-CoV-2 IgG antibody response titres and clinical characteristics of the disease. A total 92 patients were included in the study. Median age was 52 years; majority were male and middle or old aged. About $48 \%$ patients required hospitalization and $38.3 \%$ had moderate CT severity score. Positive SARS-CoV-2-IgG was detected in all patients except one. On comparing the antibody titres among various subgroups of COVID-19 recovered patients, old age was the only factor associated with statistically significant higher antibody response (28 $\mathrm{AU} / \mathrm{ml}$ for age $<35$ years, $53 \mathrm{AU} / \mathrm{ml}$ for age group 35-60, and 71 $\mathrm{AU} / \mathrm{ml}$ for age group $>60$ years, $\mathrm{p}=0.01$ ). Severity of infection, worse CT severity scores, need of hospitalization, oxygen or ventilatory support were associated with higher antibody titres but were not statistically significant. There was a strong correlation of antibody titres when analysed for age of study population (Spearman correlation $=0.39, \mathrm{p}<0.001$ ); whereas a weak correlation (Spearman correlation $=0.03, \mathrm{p}=0.753$ ) was seen when analysed for CT severity score. Elderly patients had higher antibody titre after recovery from Covid-19 infection. Severity of disease, need of hospitalisation or oxygen/mechanical ventilation did not influence the antibody titre.
\end{abstract}

\section{Introduction}

The novel coronavirus, SARS coronavirus 2 (SARS-CoV-2) originating in Wuhan city of China in December 2019, has led to a global pandemic of coronavirus disease 2019 (COVID-19), causing a serious health challenge to the entire mankind. Despite the same family of origin as of SARS, disease severity and transmissibility are much higher for SARS-CoV-2 in terms of community spread. Worse clinical outcomes are related to imbalanced Th1 immune response, which leads to uncontrolled release of cytokines, such as interleukin 6 , that mediates progression into acute respiratory distress syndrome, multiorgan failure and death [1]. Adaptive humoral 
immunity is thought to protect from acquiring SARS-CoV-2 infection, of which neutralizing antibodies play an important role. The researchers have found that antibodies against SARS-CoV-2 were readily detected in blood and saliva. IgG levels peaked about two weeks to one month after infection, then remained stable for more than 3 months [2]. The association between clinical characteristics of disease and formation of neutralizing antibodies have not well studied. In this study we aimed to study the correlation of various factors such as age, gender, severity of disease and associated co-morbidities with antibody response in patients who have recovered from COVID-19. This may have potential implications in deciding donors for convalescent plasma therapy and assessment of levels of immunity against the virus.

\section{Materials and Methods}

A prospective study was conducted for patients recovered from clinically and PCR confirmed SARS-CoV-2 infections. Patients were recruited at the Adesh Institute of Medical Sciences and Research, Bathinda, Punjab, India on an outpatient basis from $1^{\text {st }}$ August 2020 to $28^{\text {th }}$ February 2021, between 5 weeks to 8 weeks post onset of symptoms.

Epidemiological, clinical, radiological and treatment data were entered in a predesigned proforma that included information about age, sex, COVID-19 infection severity, CT (computed tomography chest) severity score, associated co-morbidities and requirement of hospitalisation, oxygen or mechanical ventilation (invasive or noninvasive) during management of COVID-19 infection. Patients were categorised as suffering from mild infection if they had mild symptoms like fever, cough, sore throat, nasal congestion, malaise, headache but without evidence of breathlessness or hypoxia (nor-

Table 1. Demographic and baseline characteristics of COVID-19 recovered patients.

\begin{tabular}{llr} 
Variable & Sub-groulp & n (\%) \\
Age (in years) & $<35$ & $17(18.5)$ \\
& $36-60$ & $41(44.6)$ \\
Sex & $>60$ & $34(36.9)$ \\
& Male & $64(69.6)$ \\
COVID-19 infection severity & Female & $28(30.4)$ \\
\hline Required hospitalization & Moderate & $47(51.1)$ \\
& Severe & $19(20.6)$ \\
Required oxygen & Yes & $26(28.3)$ \\
& No & $44(47.8)$ \\
Required high-flow oxygen or & Yes & $48(52.2)$ \\
mechanical ventilation & No & $42(45.7)$ \\
CT severity score & Yes & $50(54.3)$ \\
& No & $18(19.6)$ \\
& Mild & $74(80.4)$ \\
\hline Co-morbidities & Moderate & $24(32.9)$ \\
& Severe & $28(38.3)$ \\
& No co-morbidity & $21(28.8)$ \\
& Diabetes & $55(59.8)$ \\
& Hypertension & $22(23.9)$ \\
& Heart disease & $6(22.8)$ \\
& Respiratory disease & $3(3.5)$ \\
& Others & $1(1.1)$ \\
\hline
\end{tabular}

mal saturation), moderate: if presence of clinical features of dyspnea and or hypoxia with $\mathrm{SpO}_{2}<94 \%$ (range 90-94\%) on room air, respiratory rate more or equal to 24 per min and severe: if in addition one of the following is present; respiratory rate $>30$ breaths $/ \mathrm{min}, \mathrm{SpO}_{2}<90 \%$ on room air, severe respiratory distress; as per COVID-19 guidelines laid by Ministry of Health and Family Welfare, India [3]. CT severity score was calculated for patients who underwent CT chest during their disease evolution and management. If patient underwent more than one CT scan during COVID-19 management, the scan showing worst involvement of lung was taken as reference for calculating CT-severity score. CT severity scoring was calculated semi-quantitively as proposed by Pan et al. [4]. Extent of anatomic involvement for each of 5 lung lobes was taken in account as follows: 0 , no involvement; $1,<5 \%$ involvement; $2,5-25 \%$ involvement; $3,26-50 \%$ involvement; 4 , $51-75 \%$ involvement; and $5,>75 \%$ involvement. The final CT severity score was calculated as sum of each individual lobar score, hence ranging from 0 to 25 . Patients with CT severity score ranging from 0-7 were categorised under mild category, 8-17 as moderate and 18-25 as severe. High flow oxygen requirement was defined if patient required oxygen inhalation via HFNC (high flow nasal cannula) or NRM (non-rebreather mask) to maintain $\mathrm{SpO}_{2}$ more than or equal to $92 \%-94 \%$.

Serum samples were sent to our laboratory for determination of SARS-CoV-2-IgG. SARS-CoV-2-IgG antibody titres were determined within one day after receipt of samples by iFlashSARS-CoV-2 IgG assay, a paramagnetic particle chemiluminescent immunoassay (Shenzhen yhlo Biotech Co., Ltd) according to the manufacturer's instructions. Antibody titres above $10 \mathrm{AU} / \mathrm{ml}$ were considered reactive and significant. Patients with asymptomatic infections and PCR non-confirmed SARS-CoV-2 infections were excluded from the study population.

The study was undertaken after receiving permission from the Institutional Research and Ethical Committee.

\section{Statistical analysis}

Data was analysed by statistical software Stata 14.0. Categorical data expressed as frequency and percentage. Quantitative variable expressed as median (IQR). The average values of antibody titres were compared among various sub groups (Demographic and clinical characteristic of the patient) by Ranksum and Kruskal Wallis H test followed by Dunn test for multiple comparison. Spearman correlation coefficient was used to find the linear relationship between Antibody titre values with age and CT severity score.

\section{Results}

A total 92 patients were included in the study. Table 1 depicts the demographic and baseline characteristics of COVID-19 recovered patients. Median age of the cohort was 52 years (range 15-94). The majority of patients were middle or old aged (44.6\% and $36.9 \%$ respectively) and were males $(69.6 \%)$. A majority suffered mild infections $(51.1 \%)$ but a significant number suffered from moderate to severe infection (48.9\%) and required hospitalization (47.8\%). About $46 \%$ patients required oxygen therapy during their stay and $19.6 \%$ required high flow oxygen or mechanical ventilation. A total of 73 patients underwent $\mathrm{CT}$ chest during their disease evaluation 
and management; a majority (38.3\%) had moderate CT severity score. Among rest of the 19 patients, all suffered from mild infections only. Although majority of patients $(59.8 \%)$ had no co-morbidities; when present, diabetes (23.9\%) and hypertension $(22.8 \%)$ constituted the most common ones. Table 2 shows comparison of antibody titres amongst the various sub-groups of COVID-19 recovered patients. Positive SARS-CoV-2-IgG was detected in all patients except one. This patient was a 32-year-old male patient, who was a diagnosed case of GPA (Granulomatous polyangiitis) and was on rituximab maintenance therapy. Median antibody titres were $61 \mathrm{AU} / \mathrm{ml}$ (range 1-135). On comparing the antibody titres among various subgroups of COVID-19 recovered patients, older age was the only factor that was associated with statistically significant higher antibody response. ( $28 \mathrm{AU} / \mathrm{ml}$ for age $<35$ years, $53 \mathrm{AU} / \mathrm{ml}$ for age group 35 60 , and $71 \mathrm{AU} / \mathrm{ml}$ for age group $>60$ years, $\mathrm{p}=0.01)$. On Dunn's Pairwise Comparison of antibody titres by age (Bonferroni), $>60$ years age group had a statistically significant higher antibody titres when compared to $<35$ years age group $(\mathrm{p}=0.007)$ or $35-60$ years age group (.035), however was not statistically significant when 35 60 years age group was compared with $<35$ years age group $(\mathrm{p}=0.434)$. Among the other factors studied presence of severe infection, requirement of hospitalization, oxygen, high flow oxygen or mechanical ventilation and worse CT severity score were associated with higher antibody titres but were not statistically significant. The highest antibody titre $(135 \mathrm{AU} / \mathrm{ml})$ of the study was obtained in the patient recovered from mild infection only. There was a strong correlation of antibody titres when analysed for age of study population (Spearman correlation $=0.39, \mathrm{p}<0.001$ ); whereas a weak correlation (Spearman correlation $=0.03, \mathrm{p}=0.753$ ) was seen when analysed for CT severity score (Figures 1 and 2).

\section{Discussion}

Due to limited understanding of immunological response in SARS CoV-2, this study was conceptualised to review the factors

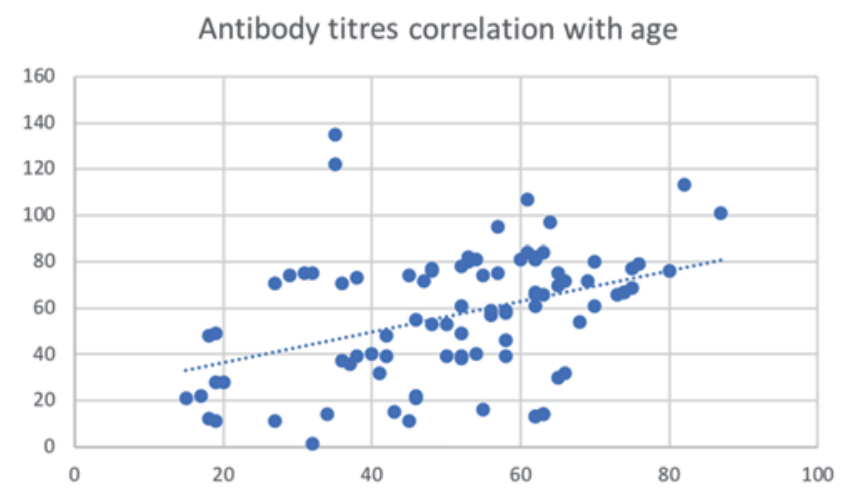

Figure 1. Antibody titres correlation with age: $\mathrm{X}$ axis depicts age and $\mathrm{Y}$ axis depicts antibody titres (Spearman correlation $=0.39$, $\mathbf{p}<\mathbf{0 . 0 0 1 )}$.

Antibody titres correlation with CT severity score

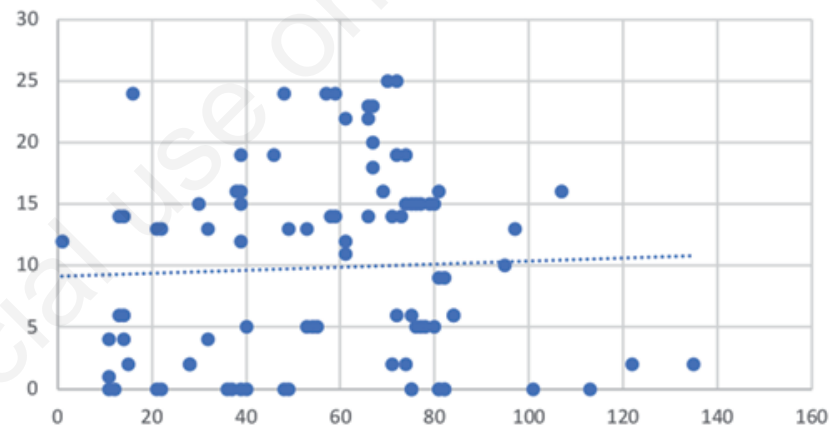

Figure 2. Antibody titres correlation with $\mathrm{CT}$ severity score: $\mathrm{X}$ axis depicts CT severity score and $\mathrm{Y}$ axis depicts antibody titres (Spearman correlation $=\mathbf{0 . 0 3}, \mathrm{p}=\mathbf{0 . 7 5 3})$.

Table 2. Antibody titres comparison among various sub-groups of COVID-19 recovered patients.

\begin{tabular}{|c|c|c|c|}
\hline Variable & Sub-group & Antibody titres (AU/ml) & p-value \\
\hline 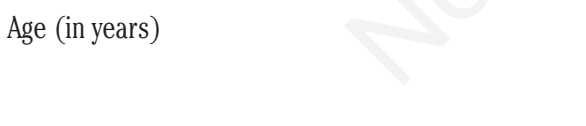 & $\begin{array}{c}<35 \\
35-60 \\
>60\end{array}$ & $\begin{array}{l}28(14-74) \\
53(39-74) \\
71(61-81)\end{array}$ & 0.01 \\
\hline Sex & $\begin{array}{l}\text { Male } \\
\text { Female }\end{array}$ & $\begin{array}{l}66.5(39-78.5) \\
55.5(28-71.5)\end{array}$ & 0.172 \\
\hline COVID-19 infection severity & $\begin{array}{c}\text { Mild } \\
\text { Moderate } \\
\text { Severe }\end{array}$ & $\begin{array}{l}54(28-80) \\
59(38-76) \\
67(48-74)\end{array}$ & 0.907 \\
\hline Required hospitalization & $\begin{array}{l}\text { Yes } \\
\text { No }\end{array}$ & $\begin{array}{l}66.5(39-74.5) \\
53.5(30-77.5)\end{array}$ & 0.589 \\
\hline Required oxygen & $\begin{array}{l}\text { Yes } \\
\text { No }\end{array}$ & $\begin{array}{l}66.5(39-74) \\
53.5(28-78)\end{array}$ & 0.556 \\
\hline Required high-flow oxygen or mechanical ventilation & $\begin{array}{l}\text { Yes } \\
\text { No }\end{array}$ & $\begin{array}{l}66.5(48-72) \\
58.5(32-78)\end{array}$ & 0.690 \\
\hline CT severity score & $\begin{array}{c}\text { Mild } \\
\text { Moderate } \\
\text { Severe }\end{array}$ & $\begin{array}{c}54(28-80) \\
61(35.5-76.5) \\
66(48-70)\end{array}$ & 0.971 \\
\hline Co-morbidities & $\begin{array}{l}\text { Yes } \\
\text { No }\end{array}$ & $\begin{array}{l}66(46-77) \\
59(32-75)\end{array}$ & 0.433 \\
\hline
\end{tabular}

Antibody titres expressed as median (inter-quartile range). 
that affect the development of neutralizing antibodies after recovery from COVID-19 infection. Detection of neutralizing antibodies after recovery from COVID-19 infection has important clinical implications. It may help to predict whether these antibodies will provide immunity against reinfection and also help in selecting appropriate donors for obtaining convalescent plasma. There are some small studies which have looked into this. The results of the current study indicate that only older age ( $>60$ years) is associated with development of higher antibody titres. All except a single patient developed antibodies. This patient was a diagnosed case of GPA and was receiving rituximab maintenance therapy. Rituximab is a B-cell depleting monoclonal antibody and there are reports of non-detection of IgG antibodies against SARS-CoV-2 in patients receiving therapy with this drug [5]. Several factors are reported to be positively correlated with higher titres of antibodies after SARS CoV-2 infection. One of the most consistent amongst these is the disease severity. Patients who are critically ill and fall under the severe category of disease have been found to have a higher titre of neutralising antibodies compared to their counterparts [6,7]. A higher baseline CT severity score has also been found to be associated with higher titres of antibodies independent of age, gender and comorbidities [8]. A plausible explanation for this fact might be "Antibody Dependent Enhancement" which is well described in SARS-CoV $[9,10]$. The high titres of anti-spike IgG was shown to shift the balance in favour of a proinflammatory state [10]. However neither clinical nor CT severity was found to be positively correlated with higher antibody titres in our study. Similarly the surrogates of clinical severity i.e. need for oxygen/mechanical ventilation, hospitalisation or comorbidities did not influence the titres of antibody after recovery from infection. This indicates that the mere correlation does not imply causality as seen in the studies quoted above. This also necessitates the need to investigate further mechanisms and pathways which influence the immunological response following infection with SARS CoV-2. However, another factor which could have influenced the results of the present study is the fact that $50 \%$ of the recruited patients belonged to the mild disease category whereas only $28 \%$ belonged to the severe category.

Factors such as varying expression of angiotensin converting enzyme receptors (ACE-2), sex hormones and innate immunity might lead to enhanced antibody expression in males compared to females. This is reflected by development of higher titres of antibodies in male patients in some studies $[6,11]$. This probably has also influenced the selection of a male donor for convalescent plasma [12]. However, our study did not find significant difference between the antibody titres between the two genders. This could potentially imply that it is not necessary to restrict plasma donation to only a single gender after an initial recovery from COVID-19 infection. The only factor which significantly influenced antibody titre in our study was a higher age group $(>60$ years) $[11,12]$. The exact influence of age and antibody titre needs further study to substantiate the causality. The strengths of the current study are an indepth analysis of association of various demographical, clinical and radiological parameters and antibody titres which have helped to partly elucidate the pattern of immunological response after recovery from COVID-19 infection. However, the criticisms could be lack of homogeneity in the distribution of patients across varying disease categories and varying time period (5-8 weeks post symptom onset) for measurement of antibody titre.

\section{Conclusions}

Elderly patients (more than 60 years) have been found to have a higher antibody titre after recovery from COVID-19 infection. Other factors such as severity of disease (clinical and radiological), hospitalisation or need for oxygen or mechanical ventilation did not influence the antibody titre.

\section{References}

1. Mojtabavi H, Saghazadeh A, Rezaei N. Interleukin-6 and severe COVID-19: a systematic review and meta-analysis. Eur Cytokine Netw 2020;31:44-9.

2. Lumley SF, Wei J, O'Donnell D, et al. The duration, dynamics and determinants of SARS-CoV-2 antibody responses in individual healthcare workers. Clin Infect Dis 2021;73:e699-709.

3. Ministry of Health [Internet]. Indian guideline for covid 19 severity - Yahoo India Search Results. Accessed: 2021 Mar 30. Available from: https://in.search.yahoo.com/search?fr= mcafee \& type $=$ E211IN826G0\&p=Ministry + of + health + Indian + guideline + for + covid $+19+$ severity

4. Pan F, Ye T, Sun P, et al. Time course of lung changes at chest CT during recovery from coronavirus disease 2019 (COVID19). Radiology 2020;295:715-21.

5. Woo MS, Steins D, Häußler V, et al. Control of SARS-CoV-2 infection in rituximab-treated neuroimmunological patients. J Neurol 2021;268:5-7.

6. Kowitdamrong E, Puthanakit T, Jantarabenjakul W, et al. Antibody responses to SARS-CoV-2 in patients with differing severities of coronavirus disease 2019. PLoS One 2020;15: e0240502.

7. Zhao J, Yuan Q, Wang H, et al. Antibody responses to SARSCoV-2 in patients with novel coronavirus disease 2019. Clin Infect Dis 2020;71:2027-34.

8. Chen W, Zhang J, Qin X, et al. SARS-CoV-2 neutralizing antibody levels are correlated with severity of COVID-19 pneumonia. Biomed Pharmacother 2020;130:110629.

9. Peiris JSM, Chu CM, Cheng VCC, et al. Clinical progression and viral load in a community outbreak of coronavirus-associated SARS pneumonia: a prospective study. Lancet 2003;361:1767-72.

10. Liu L, Wei Q, Lin Q, et al. Anti-spike IgG causes severe acute lung injury by skewing macrophage responses during acute SARS-CoV infection. JCI Insight 2019;4:e123158.

11. Klein SL, Pekosz A, Park H-S, et al. Sex, age, and hospitalization drive antibody responses in a COVID-19 convalescent plasma donor population. J Clin Inves. 2020;130:11.

12. Mehew J, Johnson R, Roberts D, Harvala H. Convalescent plasma for COVID-19: male gender, older age and hospitalisation associated with high neutralising antibody levels, England, 22 April to 12 May 2020. Euro Surveill 2020;25: 2001754. 